\title{
Structure of the solution set to impulsive functional differential inclusions on the half-line
}

\author{
Grzegorz Gabor and Agata Grudzka
}

\begin{abstract}
A topological structure of the set of solutions to impulsive functional differential inclusions on the half-line is investigated. It is shown that the solution set is nonempty, compact and, moreover, an $R_{\delta}$-set. It is proved on compact intervals and then, using the inverse limit method, obtained on the half-line.
\end{abstract}

Mathematics Subject Classification (2010). Primary 34A37; Secondary 34A60, 34K45.

Keywords. Solution set, Impulsive functional differential inclusion, Inverse systems, $R_{\delta}$-set, Topological structure.

\section{Introduction}

It is our purpose to study a topological structure of the solution set to the impulsive Cauchy problem governed by a semilinear differential inclusion on noncompact intervals.

For a fixed $\tau>0$ and a given piecewise continuous function $x:[-\tau, 0] \rightarrow$ $E$, where $E$ is a Banach space, the problem we deal with is

$$
\begin{cases}\dot{y}(t) \in A(t) y(t)+F\left(t, y_{t}\right), & \text { for a.a. } t \in[0, \infty), t \neq t_{k}, k \in \mathbb{N}, \\ y(t)=x(t), & \text { for } t \in[-\tau, 0], \\ y\left(t_{k}^{+}\right)=y\left(t_{k}\right)+I_{k}\left(y_{t_{k}}\right), & \text { for } k \in \mathbb{N}^{+},\end{cases}
$$

where $\{A(t)\}_{t \in[0, \infty)}$ is a family of linear operators in $E$ generating an evolution operator; $F$ is an upper-Carathéodory map; $y_{t}(\theta)=y(t+\theta), \theta \in[-\tau, 0] ; I_{k}$ are impulse functions, $k \in \mathbb{N}, y\left(t^{+}\right)=\lim _{s \rightarrow t^{+}} y(s)$ and the time sequence $\left(t_{k}\right)_{k \in \mathbb{N}}$ is an increasing sequence of given points in $[0, \infty)$ without accumulation points. Hence $y_{t}(\cdot)$ represents the history of the state from $t-\tau$ to the present time $t$. 
For the first time differential equations with impulses were investigated by Milman and Myshkis [1]. Impulsive differential equations and inclusions have applications in biology, economics, medicine, physics and other fields. These problems often describe phenomenas in which states are changing rapidly. One of the example is the motion of an elastic ball bouncing vertically on a surface. The moments of the impulses are in the time when the ball touches the surface and rapidly its velocity is changed. The moments of the impulse effects for the impulsive problems can be chosen in various ways: randomly, fixed beforehand, determined by the state of a system. For some recent works on impulsive differential problems, concerning the aspects we deal with, we refer to $[2-6]$.

The solution sets for differential problems often correspond with fixed point sets of multivalued operators in function spaces. In this paper we use the inverse system method, which, in studying the topological structure of fixed point sets of operators in function spaces, was initiated in [7]. This method was developed in [8] and then also in [9]. It is observed that differential problems on noncompact intervals can be reformulated as fixed point problems in Fréchet (function) spaces which are inverse limits of Banach spaces that appear when we consider these differential problems on compact intervals. Some interesting properties of fixed point sets of limit maps become very useful.

The existence of mild solutions for problem (1) has been obtained in [2]. We state and prove the compactness of the solution set for this problem. Next we prove that the set of solutions to problem (1) is an $R_{\delta}$-set.

This gives an important information from the topological point of view. The translation operator along trajectories which is often used to detect, for instance, periodic solutions, being $R_{\delta}$-valued can be checked to be an admissible (in the sense of Górniewicz) multivalued operator, and the fixed point theory methods can be applied.

The paper is organized as follows. In Sect. 2 we recall useful definitions and preliminary theorems. In the main Sect. 3 we obtain new results. In Theorem 3.4 the compactness of the solution set is proved on the half-line. The result improves Theorem 4.2 in [2], where only the existence of solutions was shown. Our proof is essentially shorter and it shows how one can effectively use the inverse systems technique. Theorems 3.5 and 3.7 are the main results of the paper. We prove the $R_{\delta}$-structure of the solution set. Note that in [4] it was shown that the solution set for the impulsive problem on compact intervals is an $R_{\delta}$-set if $F$ is a $\sigma$-Ca-selectionable multivalued map and $A(t)=A$ is the infinitesimal generator of a $C_{0}$-semigroup. The problem is that it is not clear if a sufficiently good $\sigma$-Ca-selectionability is possible in infinite dimensional spaces. In fact, as we show in the proof of Theorem 3.5, we can approximate the right-hand side of the inclusion by maps which have noncompact values and which are not $k$-set contractions. Therefore, we propose different arguments to avoid the obstacles and to prove the $R_{\delta}$-structure on compact intervals. Finally, we combine the information on a topological structure of solution sets on compact intervals with the inverse systems technique to obtain an $R_{\delta}$-structure on the half-line in Theorem 3.7. In this way we essentially 
develop some recent results in [5], where an $R_{\delta}$-structure of the solution set for the multivalued impulsive differential inclusion on the half-line is shown only in a finite dimensional case, where the compactness properties become much easier, and for the problem without any retard.

\section{Preliminaries}

Let $X, Y$ be two topological vector spaces. We denote by $P(Y)$ the family of all nonempty subsets of $Y$ and put $P_{c l}(Y)=\{A \in P(Y)$, closed $\}, P_{c l, c v}(Y)=$ $\{A \in P(Y)$, closed and convex $\}, P_{c p}(Y)=\{A \in P(Y)$, compact $\}, P_{c p, c v}(Y)=$ $\{A \in P(Y)$, compact and convex $\}$.

A multivalued map $F: X \rightarrow P(Y)$ is said to be upper semicontinuous (for short u.s.c.) if $F^{-1}(V)=\{x \in X \mid F(x) \subset V\}$ is an open subset of $X$ for every open $V \subseteq Y$. A multivalued map $F: X \rightarrow P(Y)$ is said to be lower semicontinuous (for short l.s.c.) if $F_{+}^{-1}(V)=\{x \in X \mid F(x) \cap V \neq \emptyset\}$ is an open subset of $X$ for every open $V \subseteq Y$. We say that a multivalued map $F: X \rightarrow P(Y)$ is continuous provided it is both u.s.c. and l.s.c.

Let $(X, d)$ be a metric space and $B C(X)$ denote the family of all nonempty closed bounded subsets of $X$. For given $A, B \in B C(X)$ let:

$$
d_{H}(A, B)=\inf \left\{\epsilon>0 \mid A \subset O_{\epsilon}(B) \text { and } B \subset O_{\epsilon}(A)\right\},
$$

where $O_{\epsilon}(A)=\{x \in X \mid \operatorname{dist}(x, A)<\epsilon\}$. Observe that

$$
d_{H}(A, B)=\max \left\{\sup _{a \in A} \operatorname{dist}(a, B), \sup _{b \in B} \operatorname{dist}(b, A)\right\} .
$$

The function $d_{H}: B C(X) \times B C(X) \rightarrow \mathbb{R}_{+}$is a metric on $B C(X)$ and is called the Hausdorff distance.

Let $E$ be a Banach space and $B(E)$ the family of all bounded subsets of $E$. Then the function $\beta: B(E) \rightarrow \mathbb{R}_{+}$defined by:

$\beta(A)=\inf \{r>0 \mid A$ can be covered by finitely many balls of radius $r\}$

is called the (Hausdorff) measure of noncompactness. It is monotone, nonsingular, real and regular (see [10]).

Let $I \subset \mathbb{R}$ be a compact interval, $\mu$ be a Lebesgue measure on $I$ and $E$ be a Banach space. A multivalued map $F: I \rightarrow P_{c p}(E)$ is said to be measurable (resp. weakly measurable) if for every open (resp. closed) subset $V \subset E$ the set $F^{-1}(V)$ is measurable.

A multivalued map $F: I \rightarrow P_{c p}(E)$ is said to be strongly measurable if there exists a sequence $\left\{F_{n}\right\}_{n=1}^{\infty}$ of step multivalued maps such that $d_{H}\left(F_{n}(t), F(t)\right) \rightarrow 0$ as $n \rightarrow \infty$ for $\mu$-a.e. $t \in I$.

By the symbol $L^{1}([a, b], E)$ we denote the space of all Bochner integrable functions. For simplicity of notations, we write $L^{1}([a, b])$ instead of $L^{1}([a, b], \mathbb{R})$. Let us denote by $L_{l o c}^{1}([0, \infty), E)$ the set of all Bochner integrable functions on compact subsets of $[0, \infty)$.

Let $E$ be a Banach space and $F: I \rightarrow P(E)$ be a multivalued map, where $I \subset \mathbb{R}$ is a compact interval. It is known that if $F: I \rightarrow P_{c p}(E)$ is strongly 
measurable and integrably bounded, i.e., there exists $\alpha \in L^{1}(I)$ such that

$$
\|F(t)\|:=\max \{\|y\| \mid y \in F(t)\} \leq \alpha(t)
$$

for a.e. $t \in I$, then there exists a Bochner integrable selector $f$ of $F$, i.e., $f(t) \in F(t)$ for a.e. $t \in I$.

We say that a family $\mathcal{V} \subset L^{1}(I, E)$ is integrably bounded if $V: I \rightarrow P(E)$ given by $V(t)=\{v(t) \mid v \in \mathcal{V}\}$ is integrably bounded.

Theorem 2.1. (see [10], Proposition 4.2.1.) Let $E$ be a Banach space and $\mathcal{V} \subset$ $L^{1}([a, b], E)$ be integrably bounded. Assume that the sets $V(t)$ are relatively compact for a.e. $t \in[a, b]$. Then $\mathcal{V}$ is weakly compact in $L^{1}([a, b], E)$.

We denote by $\mathcal{C}([-\tau, 0], E)$ the space of piecewise continuous functions $c:[-\tau, 0] \rightarrow E$ with finite number of discontinuity points $\left\{t_{*}\right\}$ such that $t_{*} \neq 0$ and all values

$$
c\left(t_{*}^{+}\right)=\lim _{h \rightarrow 0^{+}} c\left(t_{*}+h\right) \text { and } c\left(t_{*}^{-}\right)=\lim _{h \rightarrow 0^{-}} c\left(t_{*}+h\right)
$$

are finite. We consider the space $\mathcal{C}([-\tau, 0], E)$ with the $L^{1}$-norm, i.e.,

$$
\|c\|_{\mathcal{C}}=\int_{-\tau}^{0}\|c(t)\| d t .
$$

We do not consider the space $\mathcal{C}([-\tau, 0], E)$ with the uniform convergence norm, because it creates problems: the function $t \in[0, \infty) \rightarrow y_{t}$ is not continuous, moreover, it is not necessarily measurable (see Example 3.1, [11]). As a consequence, the multivalued superposition operator, which we will define in Sect. 3, would not be well defined. This space of delays with the integral norm was considered in [12] (see also [6]).

We denote by $P C([a, b], E)$ the space of piecewise continuous functions $c:[a, b] \rightarrow E$ with finite number of discontinuity points $\left\{t_{*}\right\}$ and such that

$$
c\left(t_{*}^{+}\right)=\lim _{h \rightarrow 0^{+}} c\left(t_{*}+h\right) \text { and } \lim _{h \rightarrow 0^{-}} c\left(t_{*}+h\right)=c\left(t_{*}\right)
$$

are finite. The space $P C([a, b], E)$ is a Banach space with the norm:

$$
\|c\|_{P C}=\sup \{\|c(t)\| \mid t \in[a, b]\}
$$

and a space of continuous functions $C([a, b], E)$ is a closed subspace of it.

We denote by $P C([a, \infty), E)$ the space of piecewise continuous functions $c:[a, \infty) \rightarrow E$ with infinite number of discontinuity points $t_{1}, t_{2}, \ldots$ such that $\lim _{n \rightarrow \infty} t_{n}=+\infty$. The values $c\left(t_{i}^{-}\right), c\left(t_{i}^{+}\right)$for $i=1,2, \ldots$ are finite and $c\left(t_{i}^{-}\right)=c\left(t_{i}\right)$. The space $P C([a, \infty), E)$ is a Fréchet space with the family of seminorms $\left\{p_{n}\right\}$ given by:

$$
p_{n}(c)=\left\|\left.c\right|_{\left[0, t_{n}\right]}\right\|_{P C}
$$

and a metric:

$$
d\left(c_{1}, c_{2}\right)=\sum_{n=1}^{\infty} \frac{1}{2^{n}} \frac{p_{n}\left(c_{1}-c_{2}\right)}{1+p_{n}\left(c_{1}-c_{2}\right)} .
$$

Let us recall that by a Fréchet space we mean a locally convex space which is metrizable and complete. Every Banach space is a Fréchet space. 
Recall that a subset $A \subset X$ is called a retract of $X$ if there exists a continuous function $r: X \rightarrow A$, such that $r(x)=x$, for every $x \in A$. $A$ is called a neighborhood retract of $X$ if there exists an open subset $U \subset X$ such that $A \subset U$ and $A$ is retract of $U$. If we have two spaces $X, Y$, then every homeomorphism $h: X \rightarrow Y$ such that $h(X)$ is a closed subset of $Y$ is called an embedding. We say that $X$ is an absolute retract (is an absolute neighborhood retract) if and only if for any space $Y$ and for any embedding $h: X \rightarrow Y$ the set $h(X)$ is a retract of $Y(h(X)$ is a neighborhood retract of $Y)$. We write $X \in A R$ (resp. $X \in A N R$ ).

A compact nonempty space is called an $R_{\delta}$-set provided there exists a decreasing sequence $\left\{A_{n}\right\}$ of compact absolute retracts such that:

$$
A=\bigcap_{n \geq 1} A_{n}
$$

Any intersection of decreasing sequence of $R_{\delta}$-sets is $R_{\delta}$.

The following characterization of $R_{\delta}$-sets, which develops the well-known Hyman's theorem [13], was shown by D. Bothe.

Theorem 2.2. (see [14]) Let $X$ be a complete metric space, $\beta$ denote the Hausdorff measure of noncompactness in $X$ and let $\emptyset \neq A \subset X$.

Then the following statements are equivalent:

(a) $A$ is an $R_{\delta}$-set,

(b) $A$ is an intersection of a decreasing sequence $\left\{A_{n}\right\}$ of closed contractible spaces with $\beta\left(A_{n}\right) \rightarrow 0$,

(c) A is compact and absolutely neighborhood contractible, i.e., A is contractible in each neighborhood in $Y \in A N R$, where $A$ is embedded.

Let us recall that an inverse system of topological spaces is a family $S=\left\{X_{\alpha}, \pi_{\alpha}^{\beta}, \Sigma\right\}$, where $\Sigma$ is a directed set ordered by the relation $\leq, X_{\alpha}$ is a topological (Hausdorff) space, for every $\alpha \in \Sigma$, and $\pi_{\alpha}^{\beta}: X_{\beta} \rightarrow X_{\alpha}$ is a continuous mapping for each two elements $\alpha, \beta \in \Sigma$ such that $\alpha \leq \beta$. Moreover, for each $\alpha \leq \beta \leq \gamma$, the following conditions should hold: $\pi_{\alpha}^{\alpha}=i d_{X_{\alpha}}$ and $\pi_{\alpha}^{\beta} \pi_{\beta}^{\gamma}=\pi_{\alpha}^{\gamma}$.

A subspace of the product $\Pi_{\alpha \in \Sigma} X_{\alpha}$ is called a limit of the inverse system $S$ and it is denoted by $\lim _{\longleftarrow} S$ whenever

$$
\lim _{\longleftarrow} S=\left\{\left(x_{\alpha}\right) \in \prod_{\alpha \in \Sigma} X_{\alpha} \mid \pi_{\alpha}^{\beta}\left(x_{\beta}\right)=x_{\alpha} \text { for all } \alpha \leq \beta\right\} .
$$

Consider two inverse systems $S=\left\{X_{\alpha}, \pi_{\alpha}^{\beta}, \Sigma\right\}$ and $S^{\prime}=\left\{Y_{\alpha^{\prime}}, \pi_{\alpha^{\prime}}^{\beta^{\prime}}, \Sigma^{\prime}\right\}$. Let us recall (see [8]) that by a multivalued map of the system $S$ into the system $S^{\prime}$, we mean a family $\left\{\sigma, \varphi_{\sigma\left(\alpha^{\prime}\right)}\right\}$ consisting of a monotone function $\sigma: \Sigma^{\prime} \rightarrow \Sigma$, i.e. $\sigma\left(\alpha^{\prime}\right) \leq \sigma\left(\beta^{\prime}\right)$ for $\alpha^{\prime} \leq \beta^{\prime}$, and of multivalued maps $\varphi_{\sigma\left(\alpha^{\prime}\right)}: X_{\sigma\left(\alpha^{\prime}\right)} \multimap Y_{\alpha^{\prime}}$ with nonempty values, defined for every $\alpha^{\prime} \in \Sigma^{\prime}$ and such that

$$
\pi_{\alpha^{\prime}}^{{\beta^{\prime}}^{\prime}} \varphi_{\sigma\left(\beta^{\prime}\right)}=\varphi_{\sigma\left(\alpha^{\prime}\right)} \pi_{\sigma\left(\alpha^{\prime}\right)}^{\sigma\left(\beta^{\prime}\right)},
$$

for each $\alpha^{\prime} \leq \beta^{\prime}$. 
A map of systems $\left\{\sigma, \varphi_{\sigma\left(\alpha^{\prime}\right)}\right\}$ induces a limit $\operatorname{map} \varphi: \lim _{\longleftarrow} S \multimap \lim _{\longleftarrow} S^{\prime}$ defined as follows:

$$
\varphi(x)=\prod_{\alpha^{\prime} \in \Sigma^{\prime}} \varphi_{\sigma\left(\alpha^{\prime}\right)}\left(x_{\sigma\left(\alpha^{\prime}\right)}\right) \cap \longleftarrow \lim _{\longleftarrow} S^{\prime}
$$

In other words, a limit map is the one such that

$$
\pi_{\alpha^{\prime}} \varphi=\varphi_{\sigma\left(\alpha^{\prime}\right)} \pi_{\sigma\left(\alpha^{\prime}\right)},
$$

for every $\alpha^{\prime} \in \Sigma^{\prime}$.

Now we summarize some useful properties of limits of inverse systems.

Proposition 2.3. (see [15]) Let $S=\left\{X_{\alpha}, \pi_{\alpha}^{\beta}, \Sigma\right\}$ be an inverse system. If, for every $\alpha \in \Sigma, X_{\alpha}$ is compact and nonempty, then $\lim _{\longleftarrow} S$ is compact and nonempty.

Proposition 2.4. (see [7]) Let $S=\left\{X_{n}, \pi_{n}^{p}, \mathbb{N}\right\}$ be an inverse system. If, for every $n \in \mathbb{N}, X_{n}$ is an $R_{\delta}$-set, then $\lim _{\longleftarrow} S$ is $R_{\delta}$, as well.

Theorem 2.5. (see [8]) Let $S=\left\{X_{\alpha}, \pi_{\alpha}^{\beta}, \Sigma\right\}$ be an inverse system and $\varphi$ : $\lim _{\longleftarrow} S \multimap \lim _{\longleftarrow} S$ be a limit map induced by a map $\left\{i d, \varphi_{\alpha}\right\}$, where $\varphi_{\alpha}$ : $X_{\alpha} \multimap X_{\alpha}$. Then the fixed point set of $\varphi$ is a limit of the inverse system generated by the sets Fix $\left(\varphi_{\alpha}\right)$. In particular, if the sets Fix $\left(\varphi_{\alpha}\right)$ are compact acyclic [resp. $\left.R_{\delta}\right]$, then it is compact acyclic [resp. $\left.R_{\delta}\right]$, as well.

\section{Structure of the solution set on noncompact intervals}

We consider the set $\triangle_{\infty}=\left\{(t, s) \in \mathbb{R}_{+} \times \mathbb{R}_{+} \mid 0 \leq s \leq t\right\}$ and the evo-

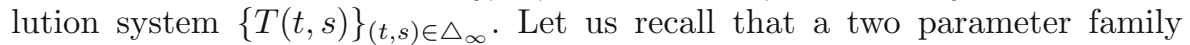
$\{T(t, s)\}_{(t, s) \in \triangle_{\infty}}$, where $T(t, s): E \rightarrow E$ is a bounded linear operator, is an evolution system if the following conditions are satisfied:

(a) $T(t, r) T(r, s)=T(t, s)$, for $0 \leq s \leq r \leq t$

(b) $T(t, t)=I d$ for $t \in[0, \infty)$,

(c) $(t, s) \rightarrow T(t, s)$ is strongly continuous on $\triangle_{\infty}$, i.e. the map $(t, s) \rightarrow$ $T(t, s) x$ is continuous on $\triangle_{\infty}$ for every $x \in E$.

We assume

$(A)^{\infty}\{A(t)\}_{t \in[0, \infty)}$ is a family of linear not necessarily bounded operators $(A(t): D(A) \subset E \rightarrow E, t \in[0, \infty), D(A)$ a dense subset of $E$ not depending on $t$ ) generating an evolution operator $T: \triangle_{\infty} \rightarrow \mathcal{L}(E)$, where $\mathcal{L}(E)$ is the space of all bounded linear operators in $E$.

The generalized Cauchy operator $G^{k}: L^{1}\left(\left[t_{k-1}, t_{k}\right], E\right) \rightarrow C\left(\left[t_{k-1}, t_{k}\right], E\right)$ is defined by

$$
G^{k} f(t)=\int_{t_{k-1}}^{t} T(t, s) f(s) d s, t \in\left[t_{k-1}, t_{k}\right] .
$$

Proposition 3.1. (see [3], Theorem 2) The generalized Cauchy operator $G^{k}$ satisfies the properties: 
(G1) there exists $c_{k} \geq 0$ such that

$$
\left\|G^{k} f(t)-G^{k} g(t)\right\| \leq c_{k} \int_{t_{k-1}}^{t}\|f(s)-g(s)\| d s, t \in\left[t_{k-1}, t_{k}\right]
$$

for every $f, g \in L^{1}\left(\left[t_{k-1}, t_{k}\right], E\right)$,

(G2) for any compact $K \subset E$ and sequence $\left(f_{n}(t)\right)_{n=1}^{\infty}, f_{n} \in L^{1}\left(\left[t_{k-1}, t_{k}\right], E\right)$, such that $\left(f_{n}(t)\right)_{n=1}^{\infty} \subset K$ for almost every $t \in\left[t_{k-1}, t_{k}\right]$, the weak convergence $f_{n} \rightarrow f_{0}$ implies the convergence $G^{k} f_{n} \rightarrow G^{k} f_{0}$.

Theorem 3.2. (see [10], Proposition 4.2.2.) Let the operator $G^{k}$ satisfy conditions (G1) and (G2) and let the set $\left\{f_{n}\right\}_{n=1}^{\infty}$ be integrably bounded with the property $\beta\left(f_{n}(t)_{n=1}^{\infty}\right) \leq \eta(t)$ for almost every $t \in\left[t_{k-1}, t_{k}\right]$, where $\eta(\cdot) \in$ $L^{1}\left(\left[t_{k-1}, t_{k}\right]\right)$. Then

$$
\beta\left(G^{k} f_{n}(t)_{n=1}^{\infty}\right) \leq 2 D \int_{t_{k-1}}^{t} \eta(s) d s, t \in\left[t_{k-1}, t_{k}\right],
$$

where $D \geq 0$ is an upper bound for norms $\|T(t, s)\|$ on $\left[t_{k-1}, t_{k}\right]$.

We consider $F:[0, \infty) \times \mathcal{C}([-\tau, 0], E) \rightarrow P_{c p, c v}(E)$ such that $(F 1)^{\infty} F(\cdot, c)$ has a strongly measurable selection for every $c \in \mathcal{C}([-\tau, 0], E)$, $(F 2)^{\infty} F(t, \cdot)$ is u.s.c. for a.e. $t \in[0, \infty)$,

$(F 3)^{\infty} \quad F$ for almost every $t \in[0, \infty)$ has at most a linear growth, i.e., there exists a function $\alpha \in L_{l o c}^{1}([0, \infty))$ such that

$$
\|F(t, c)\| \leq \alpha(t)\left(1+\|c\|_{\mathcal{C}}\right) \text { for a.e.t } \in[0, \infty),
$$

$(F 4)^{\infty}$ There exists a function $\mu \in L_{l o c}^{1}([0, \infty))$ such that

$$
\beta(F(t, D)) \leq \mu(t) \sup _{-\tau \leq \theta \leq 0} \beta(D(\theta)) \text { for a.e. } t \in[0, \infty)
$$

and for every bounded $D \subset \mathcal{C}([-\tau, 0], E), D(\theta)=\{c(\theta) \mid c \in D\}$.

The following problem on a compact interval

$$
\begin{cases}\dot{y}(t) \in A(t) y(t)+F\left(t, y_{t}\right), & \text { for a.e. } t \in\left[0, t_{m}\right], t \neq t_{k}, k<m, \\ y(t)=x(t), & \text { for } t \in[-\tau, 0], \\ y\left(t_{k}^{+}\right)=y\left(t_{k}\right)+I_{k}\left(y_{t_{k}}\right), & \text { for } k<m\end{cases}
$$

was considered in [2]. The authors proved the existence of a mild solution and that the solution set $S_{m}$ for this problem is compact.

A piecewise continuous function $y:\left[-\tau, t_{m}\right] \rightarrow E$ is a mild solution for the impulsive Cauchy problem (2) if

(a) $y(t)=T(t, 0) x(0)+\sum_{0<t_{k}<t} T\left(t, t_{k}\right) I_{k}\left(y_{t_{k}}\right)+\int_{0}^{t} T(t, s) f(s) d s, t \in\left[0, t_{m}\right]$, where $f \in L^{1}\left(\left[0, t_{m}\right], E\right), f(s) \in F\left(s, y_{s}\right)$ for almost every $s \in\left[0, t_{m}\right]$,

(b) $y(t)=x(t), t \in[-\tau, 0]$,

(c) $y\left(t_{k}^{+}\right)=y\left(t_{k}\right)+I_{k}\left(y_{t_{k}}\right), k<m$.

Let $\left[0, t_{m}\right]$ be a fixed interval on the real line. Put $\Delta_{m}=\left\{(t, s) \in\left[0, t_{m}\right] \times\right.$ $\left.\left[0, t_{m}\right] \mid s \leq t \leq t_{m}\right\}$. The authors of [2] obtained the compactness of the solution set of problem (2) under the following assumptions: 
$(A)_{m}\{A(t)\}_{t \in\left[0, t_{m}\right]}$ is a family of linear (not necessarily bounded) operators $\left(A(t): D(A) \subset E \rightarrow E, t \in\left[0, t_{m}\right], D(A)\right.$ a dense subset of $E$ not depending on $t$ ) generating an evolution operator $T: \Delta_{m} \rightarrow \mathcal{L}(E)$,

and the map $F:\left[0, t_{m}\right] \times \mathcal{C}([-\tau, 0], E) \rightarrow P_{c p, c v}(E)$ satisfies:

$(F 1)_{m} F(\cdot, c)$ has a strongly measurable selection for every $c \in \mathcal{C}([-\tau, 0], E)$, $(F 2)_{m} F(t, \cdot)$ is u.s.c. for a.e. $t \in\left[0, t_{m}\right]$,

$(F 3)_{m} F$ for almost every $t \in\left[0, t_{m}\right]$ has at most a linear growth, i.e., there exists a function $\alpha \in L^{1}\left(\left[0, t_{m}\right]\right)$ such that

$$
\|F(t, c)\| \leq \alpha(t)\left(1+\|c\|_{\mathcal{C}}\right) \text { for a.e. } t \in\left[0, t_{m}\right]
$$

$(F 4)_{m}$ There exists a function $\mu \in L^{1}\left(\left[0, t_{m}\right]\right)$ such that

$$
\beta(F(t, D)) \leq \mu(t) \sup _{-\tau \leq \theta \leq 0} \beta(D(\theta)) \text { for a.e. } t \in\left[0, t_{m}\right]
$$

and for every bounded $D \subset \mathcal{C}([-\tau, 0], E), D(\theta)=\{c(\theta) \mid c \in D\}$.

For $z \in P C\left(\left[0, t_{m}\right], E\right)$ we can define $z_{i} \in C\left(\left[t_{i}, t_{i+1}\right], E\right), i=0,1, \ldots$, $m-1$ as $z_{i}(t)=z(t)$ on $\left(t_{i}, t_{i+1}\right]$ and $z_{i}\left(t_{i}\right)=z\left(t_{i}^{+}\right)$. For every set $K \subset$ $P C\left(\left[0, t_{m}\right], E\right)$ we denote by $K_{i}, i=0,1, \ldots, m-1$ the set $K_{i}=\left\{z_{i} \mid z \in K\right\}$. It is easy to see that

Proposition 3.3. A set $K \in P C\left(\left[0, t_{m}\right], E\right)$ is relatively compact in $P C\left(\left[0, t_{m}\right], E\right)$ if and only if each set $K_{i}, i=0,1, \ldots, m-1$ is relatively compact in $C\left(\left[t_{i}, t_{i+1}\right], E\right)$.

For any $z \in P C\left(\left[0, t_{m}\right], E\right)$, or $z \in P C([0, \infty), E)$, such that $z(0)=x(0)$ we define the function $z[x]:\left[-\tau, t_{m}\right] \rightarrow E$ as

$$
z[x]=\left\{\begin{array}{l}
x(t), \mathrm{t} \in[-\tau, 0], \\
z(t), \mathrm{t} \in\left[0, \mathrm{t}_{\mathrm{m}}\right] .
\end{array}\right.
$$

where $x:[-\tau, 0] \rightarrow E$ is the function from the initial condition in (1). We denote $\Omega[x]=\{z[x] \mid z \in \Omega\}$.

For a given multivalued map $F:\left[0, t_{m}\right] \times \mathcal{C}([-\tau, 0], E) \rightarrow P_{c p, c v}(E)$ satisfying $(F 1)_{m}-(F 4)_{m}$ we consider the multivalued superposition operator $\mathcal{P}_{F}: \mathcal{D} \rightarrow P\left(L^{1}\left(\left[0, t_{m}\right], E\right)\right)$ defined as

$$
\mathcal{P}_{F}(z)=\left\{f \in L^{1}\left(\left[0, t_{m}\right], E\right) \mid f(s) \in F\left(s, z[x]_{s}\right) \text { for a.e. } s \in\left[0, t_{m}\right]\right\} .
$$

This multivalued superposition operator $\mathcal{P}_{F}$ is well defined (see e.g. [10]). Notice that the function $s \in\left[0, t_{m}\right] \rightarrow z[x]_{s} \in \mathcal{C}([-\tau, 0], E)$ is continuous.

Now, we prove the compactness of the solution set of problem (1). Note that we simultaneously obtain a nonemptiness of the solution set and our proof is essentially shorter than the one in [2].

Theorem 3.4. Let hypothesis $(A)^{\infty}$ hold, let the multivalued map $F:[0, \infty) \times$ $\mathcal{C}([-\tau, 0], E) \rightarrow P_{c p, c v}(E) \quad$ satisfy conditions $(F 1)^{\infty}-(F 4)^{\infty}$ and maps $I_{k}: \mathcal{C}([-\tau, 0], E) \rightarrow E, k \in \mathbb{N}$, be continuous. Then the solution set for problem (1) is a nonempty and compact subset of $P C([0, \infty), E)[x]$. 
Proof. Besides (1), for every $m \in \mathbb{N}^{+}$, we consider problem (2) on a compact interval $\left[0, t_{m}\right]$.

Let $C_{m}=P C\left(\left[0, t_{m}\right], E\right)[x]$. Consider the sequence of multivalued maps $\phi_{m}: C_{m} \multimap C_{m}$ as follows:

$$
\begin{array}{r}
\phi_{m}(y)(t):=\left\{T(t, 0) x(0)+\sum_{0<t_{k}<t} T\left(t, t_{k}\right) I_{k}\left(y_{t_{k}}\right)+\int_{0}^{t} T(t, s) f(s) d s, t \in\left[0, t_{m}\right]\right. \\
\left.\mid f \in L^{1}\left(\left[0, t_{m}\right], E\right), f(s) \in F\left(s, y_{s}\right) \text { for a.e. } s \in\left[0, t_{m}\right]\right\}
\end{array}
$$

for $t \in\left[0, t_{m}\right]$ and $\phi_{m}(y)(t)=x(t)$ for $t \in[-\tau, 0]$. Now we consider the projections $p_{m}^{m+1}: C_{m+1} \rightarrow C_{m}$, which are defined as follows $p_{m}^{m+1}(y)=\left.y\right|_{\left[-\tau, t_{m}\right]}$.

We have the equalities

$$
\begin{array}{r}
\phi_{m} p_{m}^{m+1}(y)(t)=\left\{T(t, 0) x(0)+\sum_{0<t_{k}<t} T\left(t, t_{k}\right) I_{k}\left(y_{t_{k}}\right)+\int_{0}^{t} T(t, s) f(s) d s\right. \\
\left.\mid t \in\left[0, t_{m}\right], f \in L^{1}\left(\left[0, t_{m}\right], E\right), f(s) \in F\left(s, y_{s}\right) \text { for a.e.s } \in\left[0, t_{m}\right]\right\} \\
p_{m}^{m+1} \phi_{m+1}(y)(t)=\left\{T(t, 0) x(0)+\sum_{0<t_{k}<t} T\left(t, t_{k}\right) I_{k}\left(y_{t_{k}}\right)+\int_{0}^{t} T(t, s) f(s) d s\right. \\
\left.\mid t \in\left[0, t_{m}\right], f \in L^{1}\left(\left[0, t_{m+1}\right], E\right), f(s) \in F\left(s, y_{s}\right) \text { for a.e.s } \in\left[0, t_{m+1}\right]\right\},
\end{array}
$$

and from the observation that

$$
\begin{aligned}
& \left\{f \in L^{1}\left(\left[0, t_{m}\right], E\right) \mid f(s) \in F\left(s, y_{s}\right) \text { for a.e. } s \in\left[0, t_{m}\right]\right\} \\
& \quad=\left\{\left.f\right|_{\left[-\tau, t_{m}\right]}, f \in L^{1}\left(\left[0, t_{m+1}\right], E\right) \mid f(s) \in F\left(s, y_{s}\right) \text { for a.e. } s \in\left[0, t_{m+1}\right]\right\}
\end{aligned}
$$

we obtain that $\phi_{m} p_{m}^{m+1}=p_{m}^{m+1} \phi_{m+1}$, so $\left\{i d, \phi_{m}\right\}$ is the map of the inverse system $\left\{C_{m}, p_{m}^{n}\right\}$. The map $\left\{i d, \phi_{m}\right\}$ induces the limit map $\phi: C \multimap C$, where $C=P C([0, \infty), E)[x]$

$$
\begin{array}{r}
\phi(y)(t)=\left\{T(t, 0) x(0)+\sum_{0<t_{k}<t} T\left(t, t_{k}\right) I_{k}\left(y_{t_{k}}\right)+\int_{0}^{t} T(t, s) f(s) d s, t \in[0, \infty)\right. \\
\left.\mid f \in L_{l o c}^{1}([0, \infty), E), f(s) \in F\left(s, y_{s}\right) \text { for a.e.s } \in[0, \infty)\right\}
\end{array}
$$

for $t \in\left[0, t_{m}\right]$ and $\phi(y)(t)=x(t)$ for $t \in[-\tau, 0]$. Note that $S:=F i x(\phi)=$ $\lim _{\longleftarrow} S_{m}$ is the solution set of problem (1). It is known (see [2], Theorem 3.7) that for every $m \geq 1$ the solution set $S_{m}$ to (2) is a nonempty and compact subset of $P C\left(\left[0, t_{m}\right], E\right)[x]$. Using Proposition 2.3 we see that the set $S$ is nonempty and compact. 
Now we prove the results about an $R_{\delta}$-structure of the solution set. At first we examine the case of problems on compact intervals. In the next step we deal with the problem on the half-line.

Theorem 3.5. Let $E$ be a Banach space and let hypothesis $(A)_{m}$ hold. Suppose that the multivalued map $F:\left[0, t_{m}\right] \times \mathcal{C}([-\tau, 0], E) \rightarrow P_{c p, c v}(E)$ satisfies conditions $(F 1)_{m}-(F 4)_{m}$. Moreover, assume that the maps $I_{k}: \mathcal{C}([-\tau, 0], E)$ $\rightarrow E, k \in \mathbb{N}$, are continuous and there exist constants $r_{k}>0$ such that,

$$
\beta\left(I_{k}(D)\right) \leq r_{k} \sup _{-\tau \leq \theta \leq 0} \beta(D(\theta))
$$

for every bounded $D \subset \mathcal{C}([-\tau, 0], E)$,

$$
\sum_{k=1}^{m} r_{k}<\frac{1}{B_{m}}
$$

where $B_{m}=\sup _{(t, s) \in \Delta_{m}}\|T(t, s)\|_{\mathcal{L}(E)}$.

Then the solution set for problem $(2)$ is an $R_{\delta}$-set in $P C\left(\left[0, t_{m}\right], E\right)[x]$.

Note that, since the evolution operator $T$ is strongly continuous on the compact set $\Delta_{m}$, the number $B_{m}$ is finite, that is, $B_{m}<\infty$.

Proof. We will proceed in several steps.

Step 1. Consider the non-impulsive Cauchy problem

$$
\begin{cases}\dot{y}(t) \in A(t) y(t)+F\left(t, y_{t}\right), & \text { for a.a. } t \in\left[0, t_{1}\right], \\ y(t)=x(t), & \text { for } t \in[-\tau, 0] .\end{cases}
$$

In [2] it was shown that solutions of (4) are bounded (by some $\bar{K}_{1} \geq 0$ ). We show that solutions on the interval $\left[t_{1}, t_{2}\right]$ are bounded by $\bar{K}_{2}$. To do this we consider the non-impulsive Cauchy problems

$$
\begin{cases}\dot{y}(t) \in A(t) y(t)+F\left(t, y_{t}\right), & \text { for a.a. } t \in\left[t_{1}, t_{2}\right] \\ y(t)=z^{1}(t), & \text { for } t \in\left[-\tau, t_{1}\right] \\ y\left(t_{1}^{+}\right)=z^{1}\left(t_{1}\right)+I_{1}\left(z_{t_{1}}^{1}\right) & \end{cases}
$$

Here the function $z^{1}$ is any solution on the $\left[-\tau, t_{1}\right]$. The mild solutions for $(5)$ have the forms:

$$
y(t)=T\left(t, t_{1}\right) z^{1}\left(t_{1}\right)+T\left(t, t_{1}\right) I_{1}\left(z_{t_{1}}^{1}\right)+\int_{t_{1}}^{t} T(t, s) f(s) d s,
$$

where $f \in L^{1}\left(\left[t_{1}, t_{2}\right], E\right), f(s) \in F\left(s, y_{s}\right), t \in\left[t_{1}, t_{2}\right]$.

We have

$$
\begin{aligned}
\|y(t)\| & \leq B_{m}\left\|z^{1}\left(t_{1}\right)\right\|+B_{m}\left\|I_{1}\left(z_{t_{1}}^{1}\right)\right\|+B_{m} \int_{t_{1}}^{t}\|f(s)\| d s \\
& \leq B_{m}\left(\bar{K}_{1}+\bar{R}\right)+B_{m} \int_{t_{1}}^{t} \alpha(s)\left(1+\left\|y_{t}\right\|_{\mathcal{C}}\right) d s \\
& \leq B_{m}\left(\bar{K}_{1}+\bar{R}+\|\alpha\|_{L^{1}\left[t_{1}, t_{2}\right]}\right)+B_{m} \int_{t_{1}}^{t} \alpha(s)\left(\int_{-\tau}^{0}\left\|y_{s}(\theta)\right\| d \theta\right) d s
\end{aligned}
$$


where $\bar{R}$ is a common upper bound for $\left\|I_{1}\left(z_{t_{1}}\right)\right\|$, where $z$ is any solution on $\left[-\tau, t_{1}\right]$, which exists because the solution set for (4) is compact and $I_{1}$ is continuous.

So, we have

$$
\begin{aligned}
\|y(t)\| & \leq \bar{M}_{2}+B_{m} \int_{t_{1}}^{t} \alpha(s)\left(\int_{-\tau}^{0}\left\|y_{s}(\theta)\right\| d \theta\right) d s \\
& \leq \bar{M}_{2}+B_{m} \int_{t_{1}}^{t} \alpha(s) \tau \cdot \sup _{-\tau \leq \theta \leq 0}\left\|y_{s}(\theta)\right\| d s \\
& \leq \bar{M}_{2}+B_{m} \int_{t_{1}}^{t} \alpha(s) \tau \cdot \sup _{-\tau \leq \theta \leq 0}\|y(s+\theta)\| d s \\
& \leq \bar{M}_{2}+B_{m} \int_{t_{1}}^{t} \alpha(s) \tau \cdot \sup _{s-\tau \leq \theta \leq s}\|y(\theta)\| d s \\
& \leq \bar{M}_{2}+B_{m} \int_{t_{1}}^{t} \alpha(s) \tau \cdot \sup _{-\tau \leq \theta \leq s}\|y(\theta)\| d s
\end{aligned}
$$

where $\bar{M}_{2} \geq B_{m}\left(\bar{K}_{1}+\bar{R}+\|\alpha\|_{L^{1}\left[t_{1}, t_{2}\right]}\right)$.

The right hand side is an increasing function in $t$, so we have the same estimate for all $t_{1}<r \leq t$. Therefore

$$
\sup _{t_{1}<r \leq t}\|y(r)\| \leq \bar{M}_{2}+\tau B_{m} \int_{t_{1}}^{t} \alpha(s) \sup _{-\tau \leq \sigma \leq s}\|y(\sigma)\| d s .
$$

Since $\|y(s)\| \leq \bar{M}_{2}$ for $s<t_{1}$, we obtain

$$
\sup _{-\tau<r \leq t}\|y(r)\| \leq \bar{M}_{2}+\tau B_{m} \int_{t_{1}}^{t} \alpha(s) \sup _{-\tau \leq \sigma \leq s}\|y(\sigma)\| d s .
$$

The function $\psi_{2}(t)=\sup _{-\tau<r<t}\|y(r)\|$ is piecewise continuous.

For the function

$$
v(t)=\int_{t_{1}}^{t} \alpha(s) \psi_{2}(s) d s
$$

we have $v\left(t_{1}\right)=0$ and $v^{\prime}(t)=\alpha(t) \psi_{2}(t)$.

We obtained the estimate $\psi_{2}(t) \leq \bar{M}_{2}+\tau B_{m} v(t)$, so

$$
v^{\prime}(t) \leq \alpha(t)\left(\bar{M}_{2}+\tau B_{m} v(t)\right) .
$$

Next we multiply the above inequality by $e^{-\tau B_{m} \int_{t_{1}}^{t} \alpha(s) d s}$

$$
v^{\prime}(t) e^{-\tau B_{m} \int_{t_{1}}^{t} \alpha(s) d s} \leq e^{-\tau B_{m} \int_{t_{1}}^{t} \alpha(s) d s} \alpha(t)\left(\bar{M}_{2}+\tau B_{m} v(t)\right) .
$$

Hence

$$
\left(v(t) e^{-\tau B_{m} \int_{t_{1}}^{t} \alpha(s) d s}\right)^{\prime} \leq e^{-\tau B_{m} \int_{t_{1}}^{t} \alpha(s) d s} \alpha(t) \bar{M}_{2} .
$$

We integrate both sides of this inequality from $t_{1}$ to $t_{2}$ and we obtain

$$
v\left(t_{2}\right) e^{-\tau B_{m} \int_{t_{1}}^{t_{2}} \alpha(s) d s} \leq \bar{M}_{2} \int_{t_{1}}^{t_{2}} \alpha(t) e^{-\tau B_{m} \int_{t_{1}}^{t} \alpha(s) d s} d t,
$$


so

$$
v\left(t_{2}\right) \leq K_{2},
$$

where $K_{2}=\frac{\bar{M}_{2} \int_{t_{1}}^{t_{2}} \alpha(t) e^{-\tau B_{m} \int_{t_{1}}^{t} \alpha(s) d s} d t}{e^{-\tau B_{m} \int_{t_{1}}^{t_{2}} \alpha(s) d s}}$. The function $v$ is nondecreasing, so

$$
v(t) \leq K_{2} \text { for all } t \in\left[t_{1}, t_{2}\right]
$$

hence

$$
\|y(t)\| \leq \bar{M}_{2}+\tau B_{m} K_{2}:=\bar{K}_{2} .
$$

Without any loss of generality we can assume that $\bar{K}_{2} \geq \bar{K}_{1}$. So, for every $k \geq 1$, every solution of the non-impulsive Cauchy problem

$$
\begin{cases}\dot{y}(t) \in A(t) y(t)+F\left(t, y_{t}\right), & \text { for a.a. } t \in\left[0, t_{k}\right] \\ y(t)=x(t), & \text { for } t \in[-\tau, 0] \\ y\left(t_{i}^{+}\right)=y\left(t_{i}\right)+I_{i}\left(y_{t_{i}}\right), & \text { for } i<k\end{cases}
$$

is bounded by $\bar{K}_{k} \geq \bar{K}_{k-1}$. We define a mapping $\tilde{F}:\left[0, t_{m}\right] \times \mathcal{C}([-\tau, 0], E) \rightarrow$ $P_{c p, c v}(E)$,

$$
\tilde{F}(t, c)= \begin{cases}F(t, c), & \text { if } t \in\left[0, t_{m}\right] \text { and }\|c\|_{\mathcal{C}} \leq \bar{K}_{m} \\ F\left(t, \frac{K_{m} c}{\|c\|_{\mathcal{C}}}\right), & \text { if } t \in\left[0, t_{m}\right] \text { and }\|c\|_{\mathcal{C}}>\bar{K}_{m}\end{cases}
$$

The function $r: \mathcal{C}([-\tau, 0], E) \rightarrow \operatorname{cl} B\left(0, \bar{K}_{m}\right) \subset \mathcal{C}([-\tau, 0], E)$ given by the formula $r(c)=\frac{\bar{K}_{m} c}{\|c\|_{\mathcal{C}}}$ for every $c \in \mathcal{C}([-\tau, 0], E)$ with $\|c\|_{\mathcal{C}}>\bar{K}_{m}$ and $r(c)=c$ for $c$ with $\|c\|_{\mathcal{C}} \leq \bar{K}_{m}$ is a continuous retraction of $\mathcal{C}([-\tau, 0], E)$ onto a closed ball. Therefore $\tilde{F}(t, c)=F(t, r(c))$ and $\tilde{F}$ has the same measurability and continuity properties as $F$. For every $m \geq 1$ and $t \in\left[0, t_{m}\right]$ the following inequalities hold

$$
\|\tilde{F}(t, c)\|=\|F(t, c)\| \leq \alpha(t)\left(1+\|c\|_{\mathcal{C}}\right) \leq \alpha(t)\left(1+\bar{K}_{m}\right)
$$

for every $\|c\|_{\mathcal{C}} \leq \bar{K}_{m}$, and

$$
\begin{aligned}
\|\tilde{F}(t, c)\| & =\left\|F\left(t, \frac{\bar{K}_{m} c}{\|c\|_{\mathcal{C}}}\right)\right\| \\
& \leq \alpha(t)\left(1+\left\|\frac{\bar{K}_{m} c}{\|c\|_{\mathcal{C}}}\right\|_{\mathcal{C}}\right) \leq \alpha(t)\left(1+\bar{K}_{m}\right)
\end{aligned}
$$

for every $\|c\|_{\mathcal{C}}>\bar{K}_{m}$. So we have:

$$
\|\tilde{F}(t, c)\| \leq \alpha(t)\left(1+\bar{K}_{m}\right) \equiv \psi_{m}(t) \in L^{1}\left(\left[0, t_{m}\right]\right) .
$$

Now we consider an impulsive problem for fixed $m$ with a multivalued $\operatorname{map} \tilde{F}$

$$
\begin{cases}\dot{y}(t) \in A(t) y(t)+\tilde{F}\left(t, y_{t}\right), & \text { for a.e. } t \in\left[0, t_{m}\right], t \neq t_{k}, k<m \\ y(t)=x(t), & \text { for } t \in[-\tau, 0] \\ y\left(t_{k}^{+}\right)=y\left(t_{k}\right)+I_{k}\left(y_{t_{k}}\right), & \text { for } k<m .\end{cases}
$$

Let $\tilde{S}_{m}$ be the solution set of problem (8). If $y$ is a solution of (2), i.e., $y \in S_{m}$, then $\|y\| \leq \bar{K}_{m} . F$ and $\tilde{F}$ coincide on $\operatorname{clB}\left(0, \bar{K}_{m}\right)$, so we have that $y \in \tilde{S}_{m}$. If $y \in \tilde{S}_{m}$, then we can easily see that $\|y\| \leq \bar{K}_{m}$ and $\dot{y}(t) \in A(t) y(t)+F\left(t, y_{t}\right)$ 
for a.e. $t \in\left[0, t_{m}\right], t \neq t_{k}, k<m$ and that $y(t)=x(t)$ for $t \in[-\tau, 0]$, so $y \in S_{m}$. We have $S_{m}=\tilde{S}_{m}$. Consequently, we can assume from now on, without any loss of generality, that

$\left(F 3^{\prime}\right)_{m}\|F(t, c)\| \leq \psi_{m}(t)$ for every $t \in\left[0, t_{m}\right]$, where $\psi_{m}(t) \in L^{1}\left(\left[0, t_{m}\right]\right)$.

Step 2. Now we prove that there exists a sequence of multivalued maps $\left\{G_{n}\right\}_{n=1}^{\infty}, G_{n}:\left[0, t_{m}\right] \times \mathcal{C}([-\tau, 0], E) \rightarrow P_{c l, c v}(E)$ such that:

(i) each multivalued map $G_{n}(t, \cdot): \mathcal{C}([-\tau, 0], E) \rightarrow P_{c l, c v}(E), n \geq 1$ is continuous for a.e. $t \in\left[0, t_{m}\right]$,

(ii) $F(t, c) \subset \cdots \subset G_{n+1}(t, c) \subset G_{n}(t, c) \subset \overline{\operatorname{conv}} F\left(t, B_{3 d_{n}}(c)\right), n \geq 1$,

(iii) $F(t, c)=\bigcap_{n \geq 1} G_{n}(t, c)$,

(iv) for each $n \geq 1$ there exists a selection $g_{n}:\left[0, t_{m}\right] \times \mathcal{C}([-\tau, 0], E) \rightarrow E$ of $G_{n}$, such that $g_{n}(\cdot, c)$ is measurable and $g_{n}(t, \cdot)$ is locally Lipschitz.

Consider the sequence $d_{n}=\frac{1}{3^{n}}, n \geq 1$. Let us cover $\mathcal{C}([-\tau, 0], E)$ by the open balls $\left\{B_{d_{n}}(c)\right\}_{c \in P C([-\tau, 0], E)}$. Since the space $\mathcal{C}([-\tau, 0], E)$ is metric, there exists a locally finite refinement $\left\{V_{j}\right\}_{j \in J}$ of the cover $\left\{B_{d_{n}}(c)\right\}_{c \in \mathcal{C}([-\tau, 0], E)}$, Now, we can associate a locally Lipschitz partition of unity $\left\{p_{j}\right\}_{j \in J}$ subordinated to the open covering $\left\{V_{j}\right\}_{j \in J}$. For every $j \in J$ let $c_{j}$ be such that $V_{j} \subset B_{d_{n}}\left(c_{j}\right)$ and define

$$
G_{n}(t, c)=\sum_{j \in J} p_{j}(c) \cdot \overline{\operatorname{conv}} F\left(t, B_{2 d_{n}}\left(c_{j}\right)\right) .
$$

To prove (ii) and (iii) note that $p_{j}(c)>0$ implies that $c \in V_{j} \subset B_{d_{n}}\left(c_{j}\right)$, hence $B_{2 d_{n}}\left(c_{j}\right) \subset B_{3 d_{n}}(c)$ and therefore

$$
F(t, c) \subset G_{n}(t, c) \subseteq \overline{\operatorname{conv}} F\left(t, B_{3 d_{n}}(c)\right) .
$$

Now we prove that $F(t, c) \supset \bigcap_{n \geq 1} G_{n}(t, c)$, because is obvious.

Let $U$ be an open and convex set such that $F(t, c) \subset U$. Then from u.s.c. there exists $\delta>0$ such that, if $d(c, \bar{c})<\delta$, then $F(t, \bar{c}) \subset U$. Hence, if $3 d_{n}<\delta$ for almost every $n$, then

$$
F\left(t, B_{3 d_{n}}(c)\right) \subset U \Longrightarrow \overline{\operatorname{conv}} F\left(t, B_{3 d_{n}}(c)\right) \subset \overline{\operatorname{conv}} U \subset \bar{U} .
$$

Let $n_{0}$ be such that $3 d_{n_{0}}<\delta$.

$$
\bigcap_{n \geq 1} G_{n}(t, c) \subset G_{n_{0}}(t, c) \subseteq \overline{\operatorname{conv}} F\left(t, B_{3 d_{n_{0}}}(c)\right) \subset \bar{U} .
$$

Since $U$ was arbitrary, we have $\bigcap_{n \geq 1} G_{n}(t, c) \subset \bigcap_{\mathbb{U}} \bar{U}=F(t, c)$, where $\mathbb{U}$ denotes a family of all open and convex subsets $U$ such that $F(t, c) \subset U$. To prove (iv) we take, for every $c_{j}, j \in J$, a measurable selection $g_{j}$ of the multivalued map $F\left(\cdot, c_{j}\right)$ and define $g_{n}:\left[0, t_{m}\right] \times \mathcal{C}([-\tau, 0], E) \rightarrow E$ as

$$
g_{n}(t, c)=\sum_{j \in J} p_{j}(c) \cdot g_{j}(t)
$$


Step 3. Now we consider the differential problem:

$$
\begin{cases}\dot{y}(t) \in A(t) y(t)+G_{n}\left(t, y_{t}\right), & \text { for a.e. } t \in\left[0, t_{m}\right], t \neq t_{k}, k<m \\ y(t)=x(t), & \text { for } t \in[-\tau, 0] \\ y\left(t_{k}^{+}\right)=y\left(t_{k}\right)+I_{k}\left(y_{t_{k}}\right), & \text { for } k<m .\end{cases}
$$

Let $S_{m}^{n}$ denote the solution set of problem (9).

We show that each sequence $\left\{y_{n}\right\}$ such that $y_{n} \in S_{m}^{n}$ for all $n \geq 1$ has a convergent subsequence $y_{n_{k}} \rightarrow y \in S_{m}$.

At first we notice that $y_{n}(t)=x(t)$ for every $t \in[-\tau, 0]$ and

$$
y_{n}(t)=T(t, 0) x(0)+\sum_{0<t_{k}<t} T\left(t, t_{k}\right) I_{k}\left(y_{n_{t_{k}}}\right)+\int_{0}^{t} T(t, s) f_{n}(s) d s
$$

for $t \in\left[0, t_{m}\right]$, where $f_{n} \in L^{1}\left(\left[0, t_{m}\right], E\right)$ is such that $f_{n}(s) \in F\left(s, y_{n_{s}}\right)$ for almost every $s \in\left[0, t_{m}\right]$.

Let $R>B_{m}=\sup _{(t, s) \in \Delta_{m}}\|T(t, s)\|_{\mathcal{L}(E)}$ be such that $\sum_{k=1}^{m} r_{k}+\frac{1}{R}<$ $\frac{1}{B_{m}}$. We know that for every bounded linear operator $S: E \rightarrow E$ we have the property: $\beta(S \Omega) \leq\|S\| \beta(\Omega)$ for every $\Omega \in B(E)$. From this property (here $S=T(t, s)$ ) we have:

$$
\beta\left(\left\{T(t, s) f_{n}(s)\right\}_{n \geq 1}\right) \leq B_{m} \beta\left(\left\{f_{n}(s)\right\}_{n \geq 1}\right) .
$$

For any $p \geq 1$ we obtain

$$
\begin{aligned}
\beta\left(\left\{f_{n}(s)\right\}_{n \geq 1}\right) & =\beta\left(\left\{f_{n}(s)\right\}_{n \geq p}\right) \leq \beta\left[F\left(\{s\} \times B\left(\left\{y_{n_{s}}\right\}_{n \geq p}, 3 d_{p}\right)\right)\right] \\
& \leq \mu(s) \cdot \sup _{-\tau \leq \theta \leq 0} \beta\left(B\left(\left\{y_{n}(s+\theta)\right\}_{n \geq p}+3 d_{p}\right)\right) \\
& \leq \mu(s)\left[\max \left(\sup _{-\tau \leq \sigma \leq 0} \beta(\{x(\sigma)\}), \sup _{0 \leq \sigma \leq s} \beta\left(\left\{y_{n}(\sigma)\right\}_{n \geq p}\right)\right)+3 d_{p}\right] \\
& =\mu(s)\left(\bar{\rho}(s)+3 d_{p}\right),
\end{aligned}
$$

where $\bar{\rho}(s)=\sup _{0 \leq \sigma \leq s} \beta\left(\left\{y_{n}(\sigma)\right\}_{n \geq 1}\right)$. We have

$$
\begin{aligned}
& \beta\left(\left\{\sum_{0<t_{k}<t} T\left(t, t_{k}\right) I_{k}\left(y_{n_{t_{k}}}\right)\right\}_{n \geq 1}\right)=\beta\left(\left\{\sum_{0<t_{k}<t} T\left(t, t_{k}\right) I_{k}\left(y_{n_{t_{k}}}\right)\right\}_{n \geq p}\right) \\
& \leq \sum_{0<t_{k}<t}\left\|T\left(t, t_{k}\right)\right\| \cdot \beta\left(\left\{I_{k}\left(y_{n_{t_{k}}}\right)\right\}_{n \geq p}\right) \\
& \leq B_{m} \sum_{0<t_{k}<t} r_{k} \sup _{-\tau \leq \theta \leq 0} \beta\left(\left\{y_{n_{t_{k}}}(\theta)\right\}_{n \geq p}\right) \\
& \leq B_{m} \sum_{0<t_{k}<t} r_{k} \cdot \max \left(\sup _{-\tau \leq \sigma \leq 0} \beta(\{x(\sigma)\}), \sup _{0 \leq \sigma \leq t_{k}} \beta\left(\left\{y_{n}(\sigma)\right\}_{n \geq 1}\right)\right) \\
& =B_{m} \sum_{0<t_{k}<t} r_{k} \cdot \sup _{0 \leq \sigma \leq t_{k}} \beta\left(\left\{y_{n}(\sigma)\right\}_{n \geq 1}\right) \\
& =B_{m} \sum_{0<t_{k}<t} r_{k} \cdot \bar{\rho}\left(t_{k}\right) .
\end{aligned}
$$


Now

$$
\begin{aligned}
& \beta\left(\left\{y_{n}(t)\right\}_{n \geq 1}\right) \\
& \quad=\beta\left(\left\{T(t, 0) x(0)+\sum_{0<t_{k}<t} T\left(t, t_{k}\right) I_{k}\left(y_{n_{t_{k}}}\right)+\int_{0}^{t} T(t, s) f_{n}(s) d s\right\}_{n \geq 1}\right) \\
& \quad \leq B_{m} \sum_{0<t_{k}<t} r_{k} \cdot \bar{\rho}\left(t_{k}\right)+2 B_{m} \int_{0}^{t} \mu(s)\left(\bar{\rho}(s)+3 d_{p}\right) d s
\end{aligned}
$$

for every $p \geq 1$. Since $d_{p} \searrow 0$ as $p \rightarrow \infty$, we obtain

$$
\begin{aligned}
\bar{\rho}(t) & \leq B_{m} \sum_{0<t_{k}<t} r_{k} \cdot \bar{\rho}\left(t_{k}\right)+2 B_{m} \int_{0}^{t} \mu(s) \bar{\rho}(s) d s \\
& \leq\left(1-\frac{B_{m}}{R}\right) \bar{\rho}(t)+2 B_{m} \int_{0}^{t} \mu(s) \bar{\rho}(s) d s
\end{aligned}
$$

and, consequently,

$$
\frac{B_{m}}{R} \bar{\rho}(t) \leq 2 B_{m} \int_{0}^{t} \mu(s) \bar{\rho}(s) d s .
$$

Thus

$$
\bar{\rho}(t) \leq 2 R \int_{0}^{t} \mu(s) \bar{\rho}(s) d s .
$$

By the Gronwall inequality we get $\bar{\rho}(t)=0$ and, as a consequence,

$$
\beta\left(\left\{y_{n}(t)\right\}_{n \geq 1}\right)=0 .
$$

This also implies that $\beta\left(\left\{f_{n}(s)\right\}_{n \geq 1}\right)=0$.

For $t \leq t^{\prime}$ in $\left[0, t_{1}\right]$ we have

$$
\left\|y_{n}\left(t^{\prime}\right)-y_{n}(t)\right\| \leq \int_{t}^{t^{\prime}} B_{1} \psi_{m}(s) d s,
$$

so, the sequence $\left\{y_{n}\right\}$ is equicontinuous. This implies the existence of a subsequence $\left\{y_{n_{l}}\right\}$ which is convergent on $\left[0, t_{1}\right]$.

Define $y_{n_{l}}^{1}(t)=T(t, 0) x(0)+T\left(t, t_{1}\right) I_{1}\left(y_{n_{t_{1}}}\right)+\int_{0}^{t} T(t, s) f_{n_{l}}(s) d s$ for $t \geq t_{1}$. Notice that $y_{n_{l}}^{1}\left(t_{1}^{+}\right)=y_{n_{l}}\left(t_{1}\right)$ for every $l \geq 1$. For $t \leq t^{\prime}$ in $\left[t_{1}, t_{2}\right]$ we have, as before,

$$
\left\|y_{n_{l}}^{1}\left(t^{\prime}\right)-y_{n_{l}}^{1}(t)\right\| \leq \int_{t}^{t^{\prime}} B_{2} \psi_{m}(s) d s
$$

and $\left\{y_{n_{l}}^{1}\right\}$ is equicontinuous. So, we can choose a convergent subsequence $\left\{y_{n_{l_{s}}}^{1}\right\}$ on $\left[t_{1}, t_{2}\right]$. We glue functions

$$
y_{n_{l_{s}}}(t)=\left\{\begin{array}{l}
y_{n_{l}}, \text { for } t \leq t_{1} \\
y_{n_{l_{s}}^{1}}, \text { for } t>t_{1}
\end{array} .\right.
$$

We proceed up to $m$ and find a convergent subsequence of $\left\{y_{n}\right\}$. Denote the limit by $y$. 
Since $\beta\left(\left\{f_{n}(s)\right\}_{n \geq 1}\right)=0$, we can assume, up to subsequence, that $f_{n} \rightarrow$ $f_{0} \in L^{1}\left(\left[0, t_{m}\right], E\right)$. Therefore, since the impulse maps are continuous,

$$
y(t)=T(t, 0) x(0)+\sum_{0<t_{k}<t} T\left(t, t_{k}\right) I_{k}\left(y_{t_{k}}\right)+\int_{0}^{t} T(t, s) f_{0}(s) d s .
$$

To prove that $f_{0}(s) \in F\left(s, y_{s}\right)$ for a.e. $s \in\left[0, t_{m}\right]$ it is sufficient to use the convexity and closedness of values of $F$, an upper semicontinuity of $F(t, \cdot)$ and some standard procedures based on the Mazur lemma.

Step 4. From Step 3 it follows that $\sup \left\{d\left(v, S_{m}\right) ; v \in S_{m}^{n}\right\} \rightarrow 0$ (an easy proof by contradiction). Therefore $\sup \left\{d\left(v, S_{m}\right) ; v \in \overline{S_{m}^{n}}\right\} \rightarrow 0$, as well. Hence, since $S_{m}$ is compact and $S_{m}^{n+1} \subset S_{m}^{n}, \beta\left(S_{m}^{n}\right)=\beta\left(\overline{S_{m}^{n}}\right) \searrow 0$ as $n \rightarrow \infty$ and $S_{m}=\bigcap_{n=1}^{\infty} \overline{S_{m}^{n}}$.

Step 5. We show, what is sufficient to finish the proof, that $\overline{S_{m}^{n}}$ is contractible for every $n \geq 1$.

Fix $\bar{y} \in \overline{S_{m}^{n}}$. We divide the interval $[0,1]$ on $m$ parts, so we have $0<\frac{1}{m}<$ $\frac{2}{m}<\cdots<\frac{m-1}{m}<1$. Let $r \in\left(0, \frac{1}{m}\right]$. We consider the problem:

$$
\begin{cases}\dot{y}(t)=A(t) y(t)+g_{n}\left(t, y_{t}\right), & \text { for a.e. } t \in\left[t_{m}-m r\left(t_{m}-t_{m-1}\right), t_{m}\right], \\ y(t)=\bar{y}(t), & \text { for } t \in\left[-\tau, t_{m}-m r\left(t_{m}-t_{m-1}\right)\right], \quad(10) \\ y\left(t_{m-1}\right)=\bar{y}\left(t_{m-1}\right)+I_{m-1}\left(\bar{y}_{t_{m-1}}\right) . & \end{cases}
$$

Here $g_{n}$ is a measurable-locally Lipschitz selection of $G_{n}$ from Step 2.

Let $\tilde{y}_{n, r}^{m}$ denote the unique solution of this problem. Then the function $y_{n, r}^{m}$ defined as:

$$
y_{n, r}^{m}(t)= \begin{cases}\bar{y}(t), & \mathrm{t} \in\left[0, t_{\mathrm{m}}-m r\left(t_{m}-t_{m-1}\right)\right] \\ \tilde{y}_{n, r}^{m}(t), & t \in\left(t_{m}-m r\left(t_{m}-t_{m-1}\right), t_{m}\right],\end{cases}
$$

satisfies $y_{n, r}^{m} \in \overline{S_{m}^{n}}$.

Next for $r \in\left(\frac{1}{m}, \frac{2}{m}\right]$ we consider the problem:

$$
\left\{\begin{array}{l}
\dot{y}(t)=A(t) y(t)+g_{n}\left(t, y_{t}\right), \\
\quad \text { for a.e. } t \in\left[t_{m-1}-m\left(r-\frac{1}{m}\right)\left(t_{m-1}-t_{m-2}\right), t_{m}\right], \\
y(t)=\bar{y}(t), \quad \text { for } t \in\left[-\tau, t_{m-1}-m\left(r-\frac{1}{m}\right)\left(t_{m-1}-t_{m-2}\right)\right], \\
y\left(t_{k}^{+}\right)=y\left(t_{k}\right)+I_{k}\left(y_{t_{k}}\right), \quad k=m-1, \\
y\left(t_{m-2}\right)=\bar{y}\left(t_{m-2}\right)+I_{m-2}\left(\bar{y}_{t_{m-2}}\right) .
\end{array}\right.
$$

Let $\tilde{y}_{n, r}^{m-1}$ denote the unique solution of this problem. Then we have $y_{n, r}^{m-1} \in$ $\overline{S_{m}^{n}}$, where:

$$
y_{n, r}^{m-1}(t)= \begin{cases}\bar{y}(t), & t \in\left[0, t_{m-1}-m\left(r-\frac{1}{m}\right)\left(t_{m-1}-t_{m-2}\right)\right], \\ \tilde{y}_{n, r}^{m-1}(t), & t \in\left(t_{m-1}-m\left(r-\frac{1}{m}\right)\left(t_{m-1}-t_{m-2}\right), t_{m}\right] .\end{cases}
$$

The last problem we consider is for $r \in\left(\frac{m-1}{m}, 1\right]$ :

$$
\begin{cases}\dot{y}(t)=A(t) y(t)+g_{n}\left(t, y_{t}\right), & \text { for a.e. } t \in\left[t_{1}-m\left(r-\frac{m-1}{m}\right) t_{1}, t_{m}\right], \\ y(t)=\bar{y}(t), & \text { for } t \in\left[-\tau, t_{1}-m\left(r-\frac{m-1}{m}\right) t_{1}\right], \\ y\left(t_{k}^{+}\right)=y\left(t_{k}\right)+I_{k}\left(y_{t_{k}}\right), & \text { for } k \in\{2,3, \cdots, m-1\} \\ y\left(t_{1}\right)=\bar{y}\left(t_{1}\right)+I_{1}\left(\bar{y}_{t_{1}}\right) . & \end{cases}
$$


Let $\tilde{y}_{n, r}^{1}$ denote the unique solution of this problem. Then the function $y_{n, r}^{1}$ defined as:

$$
y_{n, r}^{1}(t)= \begin{cases}\bar{y}(t), & t \in\left[0, t_{1}-m\left(r-\frac{m-1}{m}\right) t_{1}\right], \\ \tilde{y}_{n, r}^{1}(t), & t \in\left(t_{1}-m\left(r-\frac{m-1}{m}\right) t_{1}, t_{m}\right],\end{cases}
$$

also belongs to $\overline{S_{m}^{n}}$.

Finally we consider the following function $h_{n}:[0,1] \times \overline{S_{m}^{n}} \rightarrow \overline{S_{m}^{n}}$ :

$$
h_{n}(r, \bar{y})= \begin{cases}\bar{y}, & r=0, \\ y_{n, r}^{m}, & r \in\left(0, \frac{1}{m}\right], \\ y_{n, r}^{m-1}, & r \in\left(\frac{1}{m}, \frac{2}{m}\right], \\ \vdots & \\ y_{n, r}^{1}, & r \in\left(\frac{m-1}{m}, 1\right] .\end{cases}
$$

Here the functions $y_{n, r}^{m}, y_{n, r}^{m-1}, \ldots, y_{n, r}^{1}$ are determined by the choice of $\bar{y} \in \overline{S_{m}^{n}}$. One can show that the function $h_{n}$ is continuous applying a standard method, which use a continuous dependence on initial conditions, and remembering that the maps $I_{k}$ are continuous, when checking a continuity in $r \in\left\{\frac{i}{m} ; i=\right.$ $1, \ldots, m-1\}$. The function $h_{n}$, as continuous on $[0,1] \times \overline{S_{m}^{n}}$, is a homotopy. By definition we have $h_{n}(0, \bar{y})=\bar{y}$ and $h_{n}(1, \bar{y})=y_{n, 1}^{1}$, so $\bar{S}_{m}^{n}$ is a contractible set for every $n \in \mathbb{N}$. Therefore, from Theorem 2.2 the set $S_{m}$ is an $R_{\delta}$-set.

Theorem 3.5 enables us to examine a structure of the solution set on the half-line. The assumption (I2) will be replaced by the following: $(\mathrm{I} 2)^{\infty}$

$$
\sum_{k=1}^{m} r_{k}<\frac{1}{B_{m}}
$$

for every $m \geq 1$, where $B_{m}$ is defined in (I2) $)^{\infty}$.

Notice that, since $m \mapsto a(m)=\sum_{k=1}^{m} r_{k}$ is nondecreasing and $m \mapsto$ $b(m)=\frac{1}{B_{m}}$ is nonincreasing, (I2) ${ }^{\infty}$ often means that $r_{k}=0$ for every $k \geq 1$. In such a case all impulse maps are completely continuous. In particular, if there is an eigenvalue with a positive real part, then $r_{k}=0$ for every $k \geq 1$.

Example 3.6. Let

$$
A(t)=\left[\begin{array}{cc}
-1 & 0 \\
0 & -1
\end{array}\right] \quad \text { or } \quad A(t)=\left[\begin{array}{cc}
0 & -1 \\
1 & 0
\end{array}\right] \text { for every } t \geq 0
$$

Then $B_{m}=1$ for every $m \geq 1$, and (I2) ${ }^{\infty}$ takes the form

$$
\sum_{k=1}^{m} r_{k}<1 \text { for every } m \geq 1 \text {. }
$$

Note that assumption (I2) ${ }^{\infty}$ implies $\lim _{k \rightarrow \infty} r_{k}=0$.

Theorem 3.7. Let $E$ be a Banach space and hypothesis $(A)^{\infty}$ hold. Suppose that the multivalued map $F:[0, \infty) \times \mathcal{C}([-\tau, 0], E) \rightarrow P_{c p, c v}(E)$ satisfies conditions $(F 1)^{\infty}-(F 4)^{\infty}$. Moreover, assume that the maps $I_{k}: \mathcal{C}([-\tau, 0], E)$ 
$\rightarrow E, k \in \mathbb{N}$, are continuous and satisfy $\left(\right.$ I1) and (I2) ${ }^{\infty}$. Then the solution set for problem (1) is an $R_{\delta}$-set in $P C([0, \infty), E)[x]$.

Proof. We have proved in Theorem 3.5, that solution sets on compact intervals are $R_{\delta}$ sets (that is, for problem (2)). Next we consider an inverse system like in the proof of Theorem 3.4. Using Theorem 2.5 we obtain that the solution set of problem (1) is an $R_{\delta}$-set.

Finally we state two open questions:

1. Is Theorem 3.7 true, under sensible assumptions, for $k$-set contractive (not completely continuous) impulse maps $I_{k}$, if $\lim _{m \rightarrow+\infty} B_{m}=+\infty$ ?

2. Is Theorem 3.7 true for weakly u.s.c. weakly compact valued perturbations of $m$-accretive operators?

We believe it is possible to find some interesting positive answers and we leave the problems for further research.

\section{Acknowledgments}

The authors are indebted to the referee for his valuable comments and remarks on some very recent papers connected with the material presented above. With his helpful suggestions the paper has become more complete and familiar for the reader.

Open Access. This article is distributed under the terms of the Creative Commons Attribution Noncommercial License which permits any noncommercial use, distribution, and reproduction in any medium, provided the original author(s) and source are credited.

\section{References}

[1] Milman, V.D., Myshkis, A.: On the stability of motion in the presence of impulses. Sib. Math. J. 1, 233-237 (1960, in Russian)

[2] Benedetti, I., Rubbioni, P.: Existence of solutions on compact and non-compact intervals for semilinear impulsive differential inclusions with delay. Topol. Methods Nonlinear Anal. 32, 227-245 (2008)

[3] Cardinali, T., Rubbioni, P.: On the existence of mild solutions of semilinear evolution differential inclusions. J. Math. Anal. Appl. 308, 620-635 (2005)

[4] Djebali, S., Górniewicz, L., Ouahab, A.: Filippov-Ważewski theorems and structure of solution sets for first order impulsive semilinear functional differential inclusions. Topol. Methods Nonlinear Anal. 32, 261-312 (2008)

[5] Djebali, S., Górniewicz, L., Ouahab, A.: Topological structure of solution sets for impulsive differential inclusions in Fréchet spaces. Nonlinear Anal. 74, 2141-2169 (2011) 
[6] Obukhovskii, V., Yao, J.-C.: On impulsive functional differential inclusions with Hille-Yosida operators in Banach spaces. Nonlinear Anal. 73(6), 1715-1728 (2010)

[7] Gabor, G.: Acyclicity of solution sets of inclusions in metric spaces. Topol. Methods Nonlinear Anal. 14, 327-343 (1999)

[8] Andres, J., Gabor, G., Górniewicz, L.: Topological structure of solution sets to multivalued asymptotic problems. Z. Anal. Anwendungen 19(1), 35-60 (2000)

[9] Andres, J., Pavlačková, M.: Topological structure of solution sets to asymptotic boundary value problems. J. Differ. Equ. 248, 127-150 (2010)

[10] Kamenskii, M., Obukhovskii, V., Zecca, P.: Condensing Multivalued Maps and Semilinear Differential Inclusions in Banach Spaces. De Gruyter Ser. Nonlinear Anal. Appl., vol. 7. Walter de Gruyter, Berlin-New York (2001)

[11] Guedda, L.: Some remarks in the study of impulsive differential equations and inclusions with delay. Fixed Point Theory 12(2), 349-354 (2011)

[12] Benedetti, I., Obukhovskii, V., Zecca, P.: Controllability for impulsive semilinear functional differential inclusions with a non-compact evolution operator. Discussiones Math. Differ. Inclusions Control Optim. 31(1), 39-69 (2011)

[13] Hyman, D.M.: On decreasing sequence of compact absolute retracts. Fund. Math. 64, 91-97 (1969)

[14] Bothe, D.: Multivalued perturbations of $m$-accretive differential inclusions. Isr. J. Math. 108, 109-138 (1998)

[15] Engelking, R.: Outline of General Topology. North-Holland, Amsterdam (1968)

Grzegorz Gabor and Agata Grudzka

Faculty of Mathematics and Computer Science

Nicolaus Copernicus University

Chopina 12/18

87-100 Toruń

Poland

e-mail: ggabor@mat.umk.pl

Agata Grudzka

e-mail: agata33@mat.uni.torun.pl

Received: 6 July 2011.

Accepted: 3 December 2011. 